\title{
Point-Counterpoint
}

\section{Detrimental Antidepressant Drug-Drug Interactions: Are They Clinically Relevant?}

\author{
Sheldon Preskorn",I and Steve Werder' \\ 'Department of Psychiatry, University of Kansas School of Medicine-Wichita, Wichita, KS, USA
}

\begin{abstract}
This paper takes the position that detrimental drug-drug interactions (DDIs) involving antidepressants are clinically relevant. In doing so, it begins with a definition of the terms: drug-drug interaction, detrimental, and clinical relevance. It then discusses the issues of proof and provides an overview of the clinically relevant DDls involving antidepressants. It also gives examples involving drugs besides antidepressants based on the premise that the underlying principles are applicable regardless of the therapeutic classes of the drugs involved.

Neuropsychopharmacology (2006) 31, 1605-1612. doi:I0.1038/sj.npp. 1301070
\end{abstract}

Keywords: antidepressants; drug-drug interactions; cytochrome P450 enzymes; serious adverse effects; polypharmacy

\section{Point}

We have been asked to argue in favor of the proposition that detrimental drug-drug interactions (DDIs) involving antidepressants are clinically relevant. Of necessity, we must define the following terms: drug-drug interaction, detrimental, and clinical relevance.

We will then discuss the issues of proof and briefly overview the clinically relevant DDIs involving antidepressants. We will also use examples involving drugs besides antidepressants as the principles underlying clinically relevant DDIs are independent of the therapeutic classes of the drugs involved. Finally, we will explain why DDIs are important when considering the credentials needed for prescribing privileges.

\section{DEFINITION OF A DDI}

For the purpose of this paper, a DDI will refer to the pharmacological phenomenon whereby the presence of a second drug alters the nature, magnitude, or duration of the effect of a given dose of a first drug. An antidepressant DDI is the subset of all DDIs in which one of the two drugs involved is an antidepressant.

To expand the above definition, a change in the nature means that the observed effect seen in the patient is not something that would be expected from either drug alone

\footnotetext{
* Correspondence: Dr S Preskorn, Department of Psychiatry, University of Kansas School of Medicine-Wichita, 1010 N. Kansas, Wichita, KS 67214, USA. Tel: + I 316 29| 4767, E-mail: spreskor@kumc.edu Received 7 March 2005; revised 26 October 2005; accepted 18 November 2005

Online publication: 23 February 2006 at http://www.acnp.org/citations/ Npp022 106050 | 6 //default.pdf
}

regardless of the dose given. Examples include the serotonin syndrome resulting from the coadministration of a serotonin re-uptake inhibitor (SRI) and a monoamine oxidase inhibitor (MAOI) or a hypertensive crisis as can occur when a large amount of tyramine is consumed by a person on an MAOI.

A change observed in the magnitude or the duration of the effect of a given dose of a given drug means that the effect is one that could reasonably be expected to be produced by the first drug alone but not at the dose given. Instead, the effect is either more or less or lasts longer or shorter than would be expected for the dose given.

To further illustrate the last point, consider that the effect of any drug is determined by three variables as expressed in the following equation:

$$
\begin{aligned}
\text { Effect }= & \text { affinity for and intrinsic activity at a site of action } \\
& \times \text { drug concentration } \times \text { interindividual variance }
\end{aligned}
$$

Note: Multiplication is used in the above equation for simplicity in lieu of a more complex attempt at expressing the mathematical relationships between the three major variables and their subsets.

The first variable in this equation is the pharmacodynamics (ie what the drug does to the body). The second variable is its pharmacokinetics (ie what the body does to the drug). The third variable is the reason why all patients do not respond in exactly the same way to the same dose of the same drug. Some may be completely refractory to the beneficial effects of the drug because their illness is not affected by the mechanism of the drug. Some may be relatively or sensitive resistant to the effect of the drug and this may, in some instances, be handled by dose adjustment. 
Parenthetically, there are four major sources of biological variance among patients: genetics, age-associated changes in physiology, disease, and changes brought about by what the patient consumes and thus modifies his internal environment. Genetics are trait phenomena whereas the other three are state phenomena.

DDIs are the subset of the internal environment in which the presence of a second drug alters the response to a first drug. To further put this matter and the topic of this opinion paper into perspective, consider that most drugs treat human disease by changing the biology of the patient. The exception to this statement are anti-infectives as they are given to change the biology of the pathogen (eg bacteria) causing the infection. In terms of changing human biology, consider that $5-10 \%$ of Caucasians have a genetic polymorphism that produces functional deficiency in cytochrome P450 (CYP) 2C6 activity, an enzyme estimated to be the preferred enzymatic pathway for $30 \%$ of all clinically used drugs (Levy et al, 2000). Although the incidence of genetic deficiency is $5-10 \%$ in the high-risk Caucasian population, treatment with $40 \mathrm{mg} /$ day of fluoxetine converts $95 \%$ of individuals with genetically normal CYP 2D6 activity into phenocopies of genetic deficiency (Otton et al, 1993). This is an unintended effect, which can nevertheless have consequences when fluoxetine is coprescribed with drugs dependent on biotransformation by CYP 2D6 as a necessary step in their elimination.

DDIs can be classified as either pharmacodynamic or pharmacokinetic. A pharmacodynamic DDI is where the independent effects of the two drugs produce a change in the nature, magnitude, or duration of the effect expected from either drug alone. A pharmacokinetic DDI is where one drug alters the pharmacokinetics of a coprescribed drug (ie either its absorption, distribution, metabolism, or elimination). In a pharmacokinetic DDI, one drug may be conceptualized as the victim (ie the affected drug) and the other as the perpetrator (ie the causative agent). This nomenclature is not relevant for pharmacodynamic DDIs because both drugs are independently contributing to the DDI as a result of their separate actions on the body.

\section{DETRIMENTAL}

According to Webster, detrimental means undesirable or harmful. To further make this concept relevant to the clinical use of drugs, the authors will use the Food and Drug Administration's (FDA's) definition of an adverse event (AE): 'An adverse event is any unfavorable and unintended sign (including an abnormal laboratory finding, for example), symptom, or disease temporally associated with the use of a medicinal product, whether or not considered related to the medical product (Department of Health and Human Services et al, 2005).'

The FDA further classifies AEs on three dimensions: (a) frequency (ie likelihood that they will occur), (b) severity, and (c) seriousness. The FDA categories for frequency are (a) frequent $=$ incidence of $1 / 100$ or greater, (b) infrequent $=$ incidence of less than $1 / 100$, but greater than or equal to $1 / 1000$, and (c) rare = incidence less than $1 / 1000$. (4). Severity refers to the magnitude of the effect and is generally categorized as mild, moderate, or severe. Serious refers to whether the $\mathrm{AE}$ produces one or more of the following outcomes:

1. death (or is life threatening),

2. persistent or significant disability/incapacity,

3. hospitalization or prolongation of existing in-patient hospitalization,

4. congenital anomaly/birth defect,

5. cancer,

6. clinically significant overdose, and

7. other important medical events that require medical or surgical intervention whether that occurs in an emergency room or ambulatory surgical center to prevent death or hospitalization.

Given these definitions, severity and seriousness obviously refer to different dimensions. For example, severe dry mouth is rarely, if ever, serious, whereas even a mild myocardial infarction likely would be considered serious.

\section{CLINICAL RELEVANCE FROM A PUBLIC HEALTH PERSPECTIVE}

Clinical relevance is a judgment call balancing acceptable risk $v s$ potential benefit. The issue of DDIs has gained increasing attention as a specific risk issue over the last 15 years initially as a result of the problems encountered with terfenadine (Seldane), which eventually led to its removal from the market in the US and most other countries around the world.

To be at potential risk for a DDI, a person must be taking more than one drug. This is common in the US and the rest of the developed world. To illustrate this point, a 2004 US Public Health Survey found that $44 \%$ of all Americans 18 years of age or older took one or more prescription drug in the last 1 week, $17 \%$ took three or more, and $7 \%$ took five or more, and these numbers are approximately two to three times higher for Americans 65 years of age or older (Table 1).

Antidepressant DDIs are the subset of DDIs in which at least one of the drugs involved is an antidepressant. Antidepressant DDIs are important in part for the following reasons:

1. From a public health perspective in the US, a sizeable percentage of the US population is at risk for a DDI (Center for Disease Control, 2004). Of Americans 18 years of age or older, $10 \%$ of women and $4 \%$ of males took an antidepressant in the year from 1999 to 2000 . Moreover, these numbers are growing. Antidepressant

Table I Percentage of Americans $\geqslant 18$ and $\geqslant 65$ Years of Age on Various Levels of Prescription Medication Use ${ }^{a}$

\begin{tabular}{lcc}
\hline & $\geqslant \mathbf{1 8}$ years & $\geqslant \mathbf{6 5}$ years \\
\hline One or more drugs & $44 \%$ & $84 \%$ \\
Three or more drugs & $17 \%$ & $48 \%$ \\
Five or more drugs & $7 \%$ & $\mathrm{NA}$ \\
\hline
\end{tabular}

${ }^{\mathrm{a}}$ Reproduced with permission of the author (Preskorn et al, 2005). 
Table 2 Multiple Medication Use in Patients Seen in the Veterans Affairs Healthcare System as a Function of Age and Antidepressant (AD) Drug Use

\begin{tabular}{lcc}
\hline & Not on an AD & On an AD \\
\hline Age $<60$ years & 2 & 5 \\
Median no. of medications & 6 & 24 \\
$\%$ on $\geqslant 8$ drugs & 62 & 83 \\
$\%$ on a unique regimen & & \\
Age $\geqslant 60$ years & 4 & 6 \\
Median no. of medications & 13 & 38 \\
$\%$ on $\geqslant 8$ drugs & 75 & 96 \\
$\%$ on a unique regimen & & \\
\hline
\end{tabular}

Numbers include antidepressants.

Reproduced with permission from Preskorn (2005).

use has tripled from 1988-1994 to 1999-2000 (the latest period for which data are available).

2. The recommended duration of treatment with antidepressants is for many months to years, during which time other drugs can be added or stopped.

3. Patients on antidepressants are frequently on other medications. In fact, antidepressant treatment is more important than age as a predictor of multiple medication use in patients seen in the Veterans Administration (VA) Healthcare System (Preskorn et al, 2005; Silkey et al, 2005).

Based on a recent survey, antidepressant use predicts more multiple medication use than does age in VA outpatients ((Table 2). In populations of 1000 or more, 62 and $96 \%$ of the VA outpatients under 60 and 60 or older, respectively, were on unique drug combinations. Admittedly, the VA patient population may be more complicated in terms of both psychiatric and medical comorbidity than many other clinical populations. Nevertheless, surveys show that most clinical populations contain a sizeable percentage who are at risk for a DDI. For example, $30-35 \%$ of patients in primary care and private outpatient psychiatric clinics are taking three or more drugs in addition to their antidepressants (Preskorn, 1998b). In summary, the above data indicate that a sizeable percentage of the US population is at risk for a DDI and the percentage at risk is increased if the patient is on an antidepressant.

Recall that most drugs are given to change the biology of the patient (Equation (1)). The above studies carried out in VA outpatients found that $83-96 \%$ of those on antidepressants are biologically unique based on just the specific combination of medications that they are receiving (Preskorn et al, 2005; Silkey et al, 2005).

The frequency and extent of multiple medication use in VA outpatients has several other implications as follows:

1. The fact that the median VA patient on an antidepressant is on five to six drugs means that they are at risk for complex DDI involving much more than the effect of one drug on another.
2. The fact that there are almost no formal DDI studies involving the combined effects of more than one drug on another means that there is little systematic data to guide clinicians as to what can be expected from these complex regimens.

3. The fact that $83-96 \%$ of VA outpatients on an antidepressant is on a unique regimen means that no single VA prescriber is likely to have much clinical experience to guide their assessment of what to expect in terms of the combined effects of the total regimen their patient is receiving.

Parenthetically, some readers might wonder whether the percentage of unique drug regimen is less of a problem than it might appear because drugs can be grouped together in classes. Although a complete discussion of this issue is beyond the scope of this paper, it has been addressed elsewhere (Preskorn et al, 2005; Silkey et al, 2005). Briefly, the answer is no. The reason is that drug classes are almost always based on only one salient pharmacological feature and most drugs can be classified in multiple ways. Drugs that fit together in a class defined by one pharmacologic feature may not fit together when viewed from another pharmacologic perspective.

To illustrate this point, consider the class of antidepressants termed serotonin selective re-uptake inhibitors (SSRIs). All of the drugs in this class have the ability to relatively selectively block the serotonin transporter protein and spare effects on other common neural mechanisms of action. Nevertheless, two of the SSRIs (ie fluoxetine and paroxetine) are substantial inhibitors of the drug-metabolizing CYP enzyme, 2D6, whereas the other four are not (Preskorn, 2003a, 2003b).

\section{CLINICAL RELEVANCE: WHAT ARE CRITERIA?}

The above facts set the stage for a discussion of how to assess the clinical relevance of DDIs. A conservative position would be to decide that only DDIs resulting in an SAE should be considered clinically relevant. Certainly, few would argue that a DDI causing an SAE is not at least potentially clinically relevant. However, some might argue that this threshold, although having high specificity, lacks sensitivity (ie too many false negatives).

A liberal approach would be to claim that any DDI resulting in any untoward or unfavorable outcome of any severity is clinically relevant. Some would argue that this approach, although highly sensitive, lacks specificity (ie too many false positives).

A position between these two poles would be that clinical relevance is determined by whether the DDI results in a change in treatment by the physician, the patient, or a third party such as the FDA. Such a change could include:

1. discontinuing one or more of the drugs involved,

2. adding another drug,

3. having an additional office visit,

4. performing additional diagnostic tests, and/or

5. removing the drug from the market.

Another criterion for clinical relevance could be that the treatment outcome is less than what could reasonably be expected. 
The problem is that DDIs can produce all of these various outcomes:

1. a multitude of different types of SAEs, such as sudden death (Ferslew et al, 1998; Preskorn, 2002; Preskorn and Baker, 1997), seizures (Spigset et al, 1997), cardiac rhythm disturbances (Richard et al, 1997; Robinson et al, 2000), serotonin syndrome (Beasley et al, 1993), malignant hypertension (Otte et al, 2003), neuroleptic malignant syndrome (Preskorn, 1997b), and delirium (Reeves et al, 2002; Stanford and Stanford, 1999),

2. the apparent worsening of the disease being treated (Ludgate et al, 1985; Preskorn, 1998a, 1997a),

3. the appearance of a new disease (Malek-Ahmadi and Allen, 1995),

4. lack of efficacy (ie patient is 'resistant' to beneficial drug effect) (Johne et al, 2002),

5. poor tolerability (ie patient is 'sensitive' to adverse drug effect) (Ahmed et al, 1993; Azaz and Danenberg, 1997; Moskowitz and Burns, 1988; Robinson et al, 2000; Sperber, 1991; Spina et al, 2002; de Leon et al, 2005), and

6 . withdrawal symptoms or drug-seeking behavior on the part of the patient (Bertschy et al, 1994).

The reason is that DDIs present as an alteration in the physiology of the patient (Equation (1)) and thus can mimic almost any clinical presentation. This can make the detection of DDIs difficult, which in turn leads to an underestimation of their apparent clinical relevance.

\section{CLINICAL RELEVANCE: WHAT PROOF IS REQUIRED?}

Recognition of the above leads inextricably to a discussion of what level and type of proof is necessary to classify a DDI as clinically relevant. The issue of proof is obviously problematic for the following reason: a high threshold (ie the occurrence of an SAE) should be easier than a low threshold (ie a less than maximum treatment outcome) from both an identification and an attribution perspective.

From an identification perspective, an SAE by definition is an event that obviously and significantly changes the health status of the patient, whereas a less than optimal outcome requires knowing what the optimal outcome could have been. From an attribution perspective, the causal link between an SAE and a DDI is often easier to establish because the development of the SAE is often temporally closely linked to the addition of the second drug. In contrast, a less than optimal outcome could be due to a host of factors and is most often attributed to the patient having an illness that is resistant to treatment.

Nevertheless, the determination of clinical relevance is even a problem when the threshold is an SAE. With the SAE, the issue is how often or how reliably does the SAE have to be produced? Is a single SAE out of 1000 instances sufficient to classify the DDI as being clinically relevant or does the DDI always have to result in an SAE?

Reference to recent actions with regard to the adverse effects of single drugs is relevant to this discussion. Merck removed its analgesic rofecoxib (Vioxx) from the market because it produced a small increase in the frequency of strokes and myocardial infarctions in patients treated with the drug for 18 months or longer compared with a parallel placebo control group. Pfizer, in contrast, kept their comparable drug celecoxib (Celebrex) on the market despite increases in strokes and myocardial infarctions in patients treated with high doses of that drug. The FDA removed the otherwise safe and effective drug terfenadine (Seldane) from the market when it was found to cause Torsade des Pointes when taken in combination with a substantial CYP 3A inhibitor such as ketoconazole (Nizoral) (De Ponti et al, 2001; Ray et al, 2004; Tamargo, 2000). An estimated 250 people died from such an interaction. Given the large number of patients treated with terfenadine, 250 deaths represent an incidence much smaller than one in a thousand. The drug nefazodone (Serzone) received a black box warning as a result of its use being associated with the occurrence of liver failure leading to death or requiring liver transplantation for every 250000 patient-years of exposure. In response to that black box, Bristol-Myers-Squibb, the manufacturer of nefazodone, decided to discontinue the active marketing of this antidepressant.

To put this matter in perspective, most psychiatrists would probably consider the simultaneous use of an MAOI and an SRI to be an unequivocal example of a clinically relevant, detrimental antidepressant DDI. Similarly, the FDA requires a class label against the use of these two pharmacodynamically defined classes of antidepressants owing to the risk of a detrimental DDI resulting in a serotonin syndrome, which can range in severity from tolerability problems to an SAE. Nevertheless, there are no systematic data on the frequency with which the combined use of these two classes of drugs led to an AE much less an SAE. In fact, some neurologists have taken a position of supporting the combined use of selegiline and SRIs as being safe and clinically useful. A paper has been published showing that this combination rarely results in a detrimental inconveitible outcome (Richard et al, 1997). In summary, there is lack of inconvertible data to make decisions about the relative risks of a DDI even when considering a virtually universally accepted contraindicated DDI: the use of an SRI and an MAOI together.

\section{CLINICAL RELEVANCE: WHY IS THERE NOT MORE FORMAL DATA ON CLINICAL RELEVANCE OF DDIs?}

Imagine proposing a study to provide empirical data about the relative risk of using an MAOI and an SRI together. Would an Institutional Review Board (IRB) approve such a study? If the study were approved, how successful would the enrollment be?

The DDI involving an SRI and an MAOI together falls into the pharmacodynamic subclass of DDIs. Studies of this type of DDI are often more difficult because of the absence of a consensually agreed-upon surrogate marker for the adverse outcome (eg a serotonin syndrome). In contrast, a change in the concentration of the affected drug is often used as a surrogate in the case of a pharmacokinetically mediated DDI. A number of studies have been carried out using this surrogate, particularly for pharmacokinetic DDIs in which the mechanism is an alteration in the activity of a drugmetabolizing enzyme such as a CYP enzyme. Nevertheless, the question remains: How does one compute a change in 
the level of a victim drug into an estimate of the relative risk that a clinically meaningful DDI can occur?

To expound on this point, consider the DDI studies already mentioned above, which have shown a reproducible and sizeable difference among SSRIs in terms of their effects on various CYP enzymes such as 2D6 (Preskorn, 2003a, b). For example, treatment with the lowest usually effective dose of fluoxetine and paroxetine produce approximately a $500 \%$ increase in the plasma concentration of coprescribed drugs, which are usually dependent on CYP 2D6-mediated biotransformation to be eliminated from the body, whereas the other four SSRIs at their lowest usually effective antidepressant dose produce increases of $100 \%$ or less. Another example would be the effect of fluvoxamine $v s$ the other SSRIs on substrates for CYP 1A2. The lowest usually effective antidepressant dose of fluvoxamine (based on the countries in which it is approved as an antidepressant) produces over a $500 \%$ increase in the level of coprescribed drugs, which are usually dependent on biotransformation mediated by this enzyme for their elimination, whereas all of the other SSRIs produce almost no discernible effect on the functional activity of this CYP enzyme (Spina et al, 2002).

Despite these remarkable and highly reproducible differences, the 'so what' question can reasonably be asked. How often do such increases in the plasma concentration of the affected drug lead to a clinically meaningful untoward outcome? The problem is that such studies are purposely carried out either using a model substrate with a large therapeutic index such that no untoward effect will occur or with such a low dose that even a five-fold increase in the levels of a coprescribed drug with a narrower therapeutic index will still not result in a serious untoward outcome (Johne et al, 2002; Levy et al, 2000).

Again, consider proposing a study to determine the relative risk of an SAE occurring as a result of such an increase at doses conventionally used in routine clinical practice as opposed to the low doses typically used in pharmacokinetic DDI studies. For example, clozapine is known to have a dose-dependent risk of seizures at $300 \mathrm{mg} /$ day; the risk is approximately $1 \%$, whereas the risk is approximately 5\% (ie five times higher) at a dose of $900 \mathrm{mg} /$ day. Moreover, the risk of seizures and even the prodrome of EEG dysrhythmia has been shown to be dependent on the plasma levels of clozapine achieved (Khan and Preskorn, 2005).

Coprescribing clozapine at a dose of $300 \mathrm{mg} / \mathrm{day}$ with the usually effective antidepressant dose of fluvoxamine will produce the plasma levels of clozapine expected at a dose of $900 \mathrm{mg} /$ day or higher (Wetzel et al, 1998). In such a situation, one could hypothesize that the seizure risk would be $5 \%$ or more rather than $1 \%$. To test this hypothesis, one could carry out a study in which individuals would be assigned to one of three groups: $300 \mathrm{mg} / \mathrm{day}$ of clozapine, plus placebo, $900 \mathrm{mg} / \mathrm{day}$ of clozapine plus placebo, or $300 \mathrm{mg} /$ day of clozapine plus fluvoxamine. Some research purists might want to also include a fluvoxamine plus placebo arm. To have adequate power to test this hypothesis, the study would require 300 individuals per treatment arm as the risk of clozapine-induced seizures due to clozapine is only $5 \%$ in patients receiving $900 \mathrm{mg} /$ day.
Such a study of course would not receive IRB approval. If it did and if it confirmed a five-fold increased risk of seizures, then most would probably conclude that this clozapine-fluvoxamine DDI is clinically relevant.

Some readers might wonder if such studies are needed on the premise that any clinically relevant DDI would be detected by clinical experience. In fact, most DDIs are initially detected by clinical experience and then confirmed by either more experience or formal study. Nevertheless, formal studies may be needed to fully understand the magnitude of the problem posed by a DDI because of the complexity of multiple medication use in clinical practice as reviewed earlier in this paper. A case in point is the recent finding that coprescription of erythromycin with drugs, which it can interact with pharmacokinetically, is associated with a five-fold increase in the risk of sudden death (Ray et al, 2004).

If it is possible to underestimate the clinical relevance of erythromycin-related DDIs, which cause to a five-fold increase in the risk of sudden death, imagine the difficulty assessing the clinical relevance of DDIs leading to an increased incidence in expected AEs or reduced efficacy. In this regard, de Leon and co-workers found that the coprescription of risperidone and a substantial CYP 2D6 inhibitor such as fluoxetine produced more than a threefold increase in the odds ratio for discontinuation of the drug owing to the development of acute extrapyramidal side effects (EPS), a rate comparable to that seen in individuals genetically deficient in CYP 2D6 activity (de Leon et al, 2005).

This finding from the de Leon study illustrates the problem of detection. Although many clinicians would probably agree that a DDI leading to the discontinuation of an otherwise effective and generally well-tolerated antipsychotic is clinically relevant, how easy would it be to detect in clinical practice? First, EPS is a dose-dependent AE with risperidone; hence, a control group would be needed as in the de Leon study to determine that the risk was increased in population on the perpetrator antidepressant (eg, a substantial CYP 2D6 inhibition). To establish the mechanism with certainty, it would be best to have patients on another antidepressant which shared all of the effects of the perpetrator antidepressant except the critical one. Some might also like to have as another comparison group patients on another drug with a different pharmacology, except for sharing the mechanism of action believed to be responsible for the offending interaction. In the de Leon study, the mechanism was CYP 2D6 inhibition, and therefore an ideal prospective study would compare four groups of patients on the same dose of risperidone: (a) a group on risperidone plus placebo, (b) a group on risperidone plus an SRI, which at usual antidepressant doses routinely produces substantial CYP 2D6 inhibition (ie fluoxetine or paroxetine), (c) a group on risperidone and an SRI, which at usual antidepressant doses does not routinely cause substantial CYP 2D6 inhibition (ie citalopram, escitalopram, sertraline, or venlafaxine), and (d) a group on risperdone and a drug which is not an SRI and not even an antidepressant but which nevertheless routinely causes CYP 2D6 inhibition such as the antifungal agent, terbenafine. To prove the mechanism was solely CYP 2D6 inhibition, the incidence of discontinuation owing to EPS 
would have to not be different in groups a and $\mathrm{c}$ whereas the incidence would be significantly higher in groups $b$ and $d$ and not different from each other. In addition, the study would have to be powered to detect what was considered a clinically meaningful increase rate of discontinuation and of course would have to receive IRB approval. In the de Leon study, there were both a negative and a positive control group: genetic CYP 2D6 EMs and genetic CYP 2D6 PMs and then the experimental group which was genetic EMs on a drug that produces substantial CYP 2D6 inhibition at usual clinical doses.

Considering the de Leon study further, it is also obvious why proper attribution of an adverse outcome in clinical practice is difficult. The outcome in de Leon's study was discontinuation of risperidone in hospitalized patients. In this setting, the reason for the discontinuation can be ascertained with some confidence. In clinical practice, most treatment with risperidone is carried out in outpatients. In this setting, the EPS will likely not occur for the first time when the patient is in the office for the clinician to see it. The clinician will only see the EPS if the patient remains on the same dose of risperidone until they come back for their appointment. That could occur with mild Parkinsonism or akathisia, but would not likely happen in the case of acute dystonia or more severe Parkinsonism or akathisia. In the latter cases, the patient is likely to discontinue risperidone, the offending antidepressant, or both. In that case, the patient may return to the clinic at their follow-up appointment and report their EPS. In this scenario, the clinician will likely attribute the event as due to the patient being 'sensitive' to risperidone, but may not understand why they are sensitive (ie the concomitant treatment with a substantial CYP 2D6 inhibitor). The patient could also experience a psychotic relapse secondary to discontinuing the risperidone, in which case they may end up in the emergency room and then admitted. In this scenario, their relapse may contribute to noncompliance without realizing that the noncompliance was due to an antidepressant-mediated DDI. Certainly, many clinicians would consider a psychotic relapse leading to hospitalization to be a clinically significant change in outcome, but they may not realize that the cause was an antidepressantmediated DDI.

\section{ARE THERE CLINICALLY RELEVANT DDIs INVOLVING ANTIDEPRESSANTS?}

The above discussion illustrates that the extent of clinically relevant DDIs involving antidepressants may be currently substantially underestimated. With this caveat in mind, there are a number that are consensually accepted. Most clinicians would agree that MAOIs lead the list of antidepressants with clinically relevant DDIs. These include serotonin syndrome resulting from the combined use of an MAOI and an SRI or some other drugs capable of increasing the release of serotonin in the brain, and hypertensive crisis resulting from the combined ingestion of an MAOI and foods rich in tyramine and perhaps to a lesser extent when used in combination with norepinephrine re-uptake pump inhibitors. These DDIs are considered so clinically relevant that MAOIs are rarely used today, and some would argue that they are underutilized even when other antidepressants have proven ineffective.

Tricyclic antidepressants (TCAs) are probably the next class of antidepressants with the most compelling evidence for clinically relevant DDIs. TCAs can be divided into two main classes: tertiary amine TCAs (eg amitriptyline) and secondary amine TCAs (eg nortriptyline, which is the $\mathrm{N}$ demethlyated metabolite of amitriptyline).

Tertiary amine TCAs have multiple actions and thus can pharmacodynamically interact in multiple ways with other drugs. Tertiary amine TCAs are serotonin and norepinephrine re-uptake pump inhibitors as they can interact with MAOIs to cause both the serotonin syndrome and hypertensive crisis, respectively. In contrast, secondary amine TCAs are relatively selective norepinephrine re-uptake inhibitors. For this reason, they can cause hypertensive crisis when used in combination with MAOIs but rarely, if ever, the serotonin syndrome.

Tertiary amine TCAs also block histamine receptors centrally and alpha-1 adrenergic receptors. As a result of the histamine blockade centrally, tertiary amine TCAs can potentiate the sedative effects of many drugs as well as alcohol. As a result of the blockade of alpha-1 adrenergic receptors, tertiary amine TCAs can potentiate the effects of many antihypertensive drugs, particularly beta-blockers (Thornton, 1979).

TCAs have a narrow therapeutic index as a result of concentration-dependent central nervous system and cardiac toxicity. Their principal biotransformation required for elimination is ring hydroxylation mediated by CYP 2D6 (Shad and Preskorn, 2000). In addition, tertiary amine TCAs are $N$-demethylated principally by CYP $1 \mathrm{~A} 2$ and $3 \mathrm{~A}$ (Shad and Preskorn, 2000). As such, they are susceptible to clinically relevant, pharmacokinetic DDIs when coprescribed with substantial inhibitors of these CYP enzymes. These include CYP 2D6 inhibitors such as bupropion fluoxetine, paroxetine, and terbenafine and CYP 1A2 inhibitors such as fluvoxamine and quinilone antibiotics, and CYP $3 \mathrm{~A}$ inhibitors such as fluvoxamine, nefazodone, some azole antifungals and some macrolide antibiotics (Ahmed et al, 1993).

Beyond the tertiary amine TCAs, there are a number of other antidepressants that inhibit the serotonin transporter protein. These include all of the SSRIs and the serotoninnorepinephrine re-uptake inhibitors duloxetine and venlafaxine. As a result, these drugs can all cause a serotonin syndrome when used in combination with MAOIs. Owing to their ability to block norepinephrine uptake, duloxetine and venlafaxine can also cause a hypertensive crisis when used in combination with MAOIs.

In addition to the above, a few antidepressants produce substantial inhibition of specific CYP enzymes at their usually effective doses. These (and the enzymes they inhibit) include bupropion (2D6), fluoxetine (2D6, 2C9/ $10)$, fluvoxamine $(1 \mathrm{~A} 2,3 \mathrm{~A})$, nefazodone $(3 \mathrm{~A})$, and paroxetine (2D6) (Shad and Preskorn, 2000, 2003a,b). These drugs can substantially elevate the levels of coprescribed drugs which are dependent on one or more of these specific CYP enzymes. As discussed above, the seriousness of a DDI will be a function of the dose of the victim drug and its therapeutic index. 


\section{DDIs AND PRESCRIBING PRIVILEGES}

There is considerable debate about the nature and extent of training needed to safely and effectively prescribe medications. This issue is often considered only with regard to using one drug in isolation. However, many patients are on multiple medications with complex potential to interact in a clinically meaningful way (Preskorn et al, 2005; Silkey et al, 2005).

The knowledge and skill necessary to avoid untoward DDIs are necessarily the same as those needed to safely and effectively prescribe medications. These include being able to

1. diagnose the patient adequately,

2. select appropriate medication(s) to treat all of the illness(es) the patient has, and

3. take into account the biological variance that distinguishes the specific patient from the usual patient in the registration trials that support the approval of drugs. This biological variance includes that created by the other medications that the patient is taking.

Thus, the issue of clinical relevance of detrimental DDIs is fundamental to the ongoing debate about prescribing privileges.

\section{SUMMARY}

The question posed for this discussion appears simple, but the answer is necessarily complex. The answer involves understanding how drugs interact, the limited amount of systematic study that has been carried out to test the clinical relevance of DDIs, and the complex nature of multiple medication use in routine clinical practice.

\section{ACKNOWLEDGEMENTS}

This article is adapted with permission from Sheldon Preskorn, MD. Drug-drug interactions: Proof of relevance (Part I). Journal of Psychiatric Practice 2005;11:116-22.

\section{REFERENCES}

*The references marked with an asterisk can be accessed in their entirety on the website, www.preskorn.com.

Ahmed I, Dagincourt PG, Miller LG, Shader RI (1993). Possible interaction between fluoxetine and pimozide causing sinus bradycardia. Can J Psychiatry 38: 62-63.

Azaz LT, Danenberg HD (1997). Tachycardia, orthostatic hypotension and profound weakness due to concomitant use of fluoxetine and nifedipine. Pharmacopsychiatry 30: 274-275.

Beasley Jr CM, Masica DN, Heiligenstein JH, Wheadon DE, Zerbe RL (1993). Possible monoamine oxidase inhibitor-serotonin uptake inhibitor interaction: fluoxetine clinical data and preclinical findings. J Clin Psychopharmacol 13: 312-320.

Bertschy G, Baumann PA, Eap CB, Baettig D (1994). Probable metabolic interaction between methadone and fluvoxamine in addict patients. Ther Drug Monit 16: 42-45.

Center for Disease Control. Almost Half of Americans Use at Least One Prescription Drug Annual Report on Nation's Health Shows. http://www.cdc.gov/od/oc/media/pressrel/r041202.htms. 4-19-2006.
De Leon J, Susce MT, Pan RM, Fairchild M, Koch WH, Wedlund PJ (2005). The CYP2D6 poor metabolizer phenotype may be associated with risperidone adverse drug reactions and discontinuation. J Clin Psychiatry 66: 15-27.

De Ponti F, Poluzzi E, Montanaro N (2001). Organising evidence on QT prolongation and occurrence of Torsades de Pointes with non-antiarrhythmic drugs: a call for consensus. Eur J Clin Pharmacol 57: 185-209.

Department of Health and Human Services and http://www.fda. gov/oc/gcp/regulations.html (2005). FDA regulations relating to good clinical practice and clinical trials. Department of Health and Human Services.

Ferslew KE, Hagardorn AN, Harlan GC, McCormick WF (1998). A fatal drug interaction between clozapine and fluoxetine. $J$ Forensic Sci 43: 1082-1085.

Johne A, Schmider J, Brockmoller J, Stadelmann AM, Stormer E, Bauer S et al (2002). Decreased plasma levels of amitriptyline and its metabolites on comedication with an extract from St John's wort (Hypericum perforatum). J Clin Psychopharmacol 22: $46-54$.

Khan AY, Preskorn SH (2005). Examining concentration-dependent toxicity of clozapine: role of therapeutic drug monitoring. J Psych Prac 11: 1-13.

Levy RH, Thummel KE, Trager WF, Hansten P, Eichelbaum M (2000). Metabolic Drug Interactions. Lippincott, Williams \& Wilkins: Philadelphia.

Ludgate J, Keating J, O’Dwyer R, Callaghan N (1985). An improvement in cognitive function following polypharmacy reduction in a group of epileptic patients. Acta Neurol Scand 71: 448-452.

Malek-Ahmadi P, Allen SA (1995). Paroxetine-molindone interaction [case report]. J Clin Psychiatry 56: 82-83.

Moskowitz H, Burns M (1988). The effects on performance of two antidepressants, alone and in combination with diazepam. Prog Neuropsychopharmacol Biol Psychiat 12: 783-792.

Otte W, Birkenhager TK, van den Broek WW (2003). Fatal interaction between tranylcypromine and imipramine. Eur Psychiatry 18: 264-265.

Otton SV, Wu D, Joffe RT, Cheung SW, Sellers EM (1993). Inhibition by fluoxetine of cytochrome P450 2D6 activity. Clin Pharmacol Ther 53: 401-409.

${ }^{\star}$ Preskorn SH (1997a). Do you believe in magic? J Pract Psychiatry Behav Health 3: 99-103.

*Preskorn SH (1997b). I don't see'em. J Pract Psychiatry Behav Health 3: 302-307.

*Preskorn SH (1998a). A message from Titanic. J Pract Psychiatry Behav Health 4: 236-242.

*Preskorn SH (1998b). Do you feel lucky? J Pract Psychiatry Behav Health 4: 37-40.

*Preskorn SH (2002). Fatal drug-drug interactions as a differential consideration in apparent suicides. J Psych Prac 8: 233-238.

*Preskorn SH (2003a). Reproducibility of the in vivo effect of the selective serotonin reuptake inhibitors on the in vivo function of cytochrome P450 2D6: an update (Part I). J Psych Prac 9: 150-158.

*Preskorn SH (2003b). Reproducibility of the in vivo effect of the selective serotonin reuptake inhibitors on the in vivo function of cytochrome P450 2D6: an update (Part II). J Psych Prac 9: 228-236.

Preskorn SH (2005). Multiple medication use in patients seen in the Veterans Affairs Healthcare System: so what? J Psych Prac 11: 46-50.

Preskorn SH, Baker B (1997). Fatality associated with combined fluoxetine-amitriptyline therapy. JAMA 277: 682.

Preskorn SH, Silkey B, Shah R, Neff M, Jones TL, Choi J et al (2005). Complexity of medication use in the Veterans Affairs Healthcare System: Part I: outpatient use in relation to age and number of prescribers. J Psych Prac 11: 5-15. 
Ray WA, Murray KT, Meredith S, Narasimhulu SS, Hall K, Stein CM (2004). Oral erythromycin and the risk of sudden death from cardiac causes. $N$ Engl J Med 351: 1089-1096.

Reeves RR, Mack JE, Beddingfield JJ (2002). Neurotoxic syndrome associated with risperidone and fluvoxamine. Ann Pharmacother 36: $440-443$.

Richard IH, Kurlan R, Tanner C, Factor S, Hubble J, Suchowersky O et al, Parkinson Study Group (1997). Serotonin syndrome and the combined use of deprenyl and an antidepressant in Parkinson's disease. Neurology 48: 1070-1077.

Robinson RF, Nahata MC, Olshefski RS (2000). Syncope associated with concurrent amitriptyline and fluconazole therapy. Ann Pharmacother 34: 1406-1409.

Shad MU, Preskorn SH (2000). Antidepressants. In: Levy RH, Thummel KE, Trager WF, Hansten PD, Eichelbaum M (eds). Metabolic Drug Interactions. Lippincott, Williams \& Wilkins: Philadelphia, pp 563-577.

Silkey B, Preskorn SH, Shah R (2005). Complexity of medication use in the Veterans Affairs Healthcare System: Part II. Antidepressant use among younger and older outpatients. $J$ Psych Prac 11: 16-26.

Sperber AD (1991). Toxic interaction between fluvoxamine and sustained release theophylline in an 11-year-old boy. Drug Safety 6: $460-462$.
Spigset O, Hedenmalm K, Dahl ML, Wiholm BE, Dahlqvist R (1997). Seizures and myoclonus associated with antidepressant treatment: assessment of potential risk factors, including CYP2D6 and CYP2C19 polymorphisms, and treatment with CYP2D6 inhibitors. Acta Psychiatr Scand 96: 379-384.

Spina E, Avenoso A, Scordo MG, Ancione M, Madia A, Gatti G et al (2002). Inhibition of risperidone metabolism by fluoxetine in patients with schizophrenia: a clinically relevant pharmacokinetic drug interaction. J Clin Psychopharmacol 22: 419-423.

Stanford BJ, Stanford SC (1999). Postoperative delirium indicating an adverse drug interaction involving the selective serotonin reuptake inhibitor, paroxetine? J Psychopharmacol 13: 313-317.

Tamargo J (2000). Drug-induced Torsades de Pointes: from molecular biology to bedside. Japanese J Pharmacol 83: $1-19$.

Thornton WE (1979). Tricyclic antidepressant and cardiovascular drug interactions. Am Fam Physician 20: 97-99.

Wetzel H, Anghelescu I, Szegedi A, Wiesner J, Weigmann H, Härtter S et al (1998). Pharmacokinetic interactions of clozapine with selective serotonin reuptake inhibitors: differential effects of fluvoxamine and paroxetine in a prospective study. J Clin Psychopharmacol 18: 2-9. 